\title{
Production Control Process using Integrated Robust Data Envelopment Analysis and Fuzzy Neural Network
}

\author{
Hadi Gholizadeh \\ Department of Industrial Engineering \\ Mazandaran University of Science and Technology, Babol, Iran \\ Hamed Fazlollahtabar \\ Department of Industrial Engineering \\ School of Engineering, Damghan University, Damghan, Iran \\ *Corresponding author: hfazl@du.ac.ir
}

(Received October 14, 2018; Accepted February 25, 2019)

\begin{abstract}
Considering the importance of the quality and responsiveness of manufacturing companies to customers, the most important principle can be considered to be the reduction of the cycle time of the production process. Because in the real world due to measurement errors and inaccuracies of information and the uncertainty of processes the concept Fuzzy is used. Therefore, in this study, the parameters for controlling the process of forming are optimized to reduce the cycle time. Since the optimization of an aspect of quality may modify other aspects, so, to overcome this problem, the multi-response method (Taguchi MRT) has been developed. Also, integrated fuzzy-neural network and data envelopment analysis is used for optimization and analysis purposes. Experimental results are done to measure the effectiveness of this approach in a manufacturing company in Iran.
\end{abstract}

Keywords- Production control; fuzzy neural network; data envelopment analysis; robust optimization.

\section{Introduction}

Forming disposable appliances is one of the important processes used to achieve optimum quality and product dimensions. In the forming process, selection of control parameters such as feeding speed, mold temperature, wind pressure and sheet thickness to achieve high quality performance is important in the minimum processing time.

Taguchi's method is an effective tool for designing an experiment that evaluates the effect of performing each process parameter on responses by performing a minimum number of experiments (Sastry et al., 2012; Fisher and Yates, 1943). Signal-to-Noise Ratio the best level of each parameter for a particular response is based on a better quality characteristic such as a lower, better, better, and better name (Nian et al., 1999). Regardless of the quality characteristics selected for a particular response, the $S / N$ ratio levels are favorable to the different factors for this particular response.

The Taguchi method is sufficient enough to address the problems of a target, but not suited to solving multi-objective problems. These types of problems are typically solved by assigning individual weight to a response based on priority. The fuzzy logical approach in this study is used to overcome all of these hypotheses and the uncertainty regime.

In order to optimize MRT problems, different methods and methods have been used in literature. In summary, the proposed approaches used to solve the problem of MRT, weighting methods 
International Journal of Mathematical, Engineering and Management Sciences

Vol. 4, No. 3, 580-590, 2019

https://dx.doi.org/10.33889/IJMEMS.2019.4.3-046

(Antony, 2001) gray relationship analysis (Muthuramalingam and Mohan, 2014) fuzzy logic (Pandey and Panda, 2014) principal components analysis (PCA) (Madhavi et al., 2017) Genetic algorithm (Huang et al., 2016) graph and matrix theory (Rao et al., 2018) artificial intelligence (Sibalija and Majstorovic, 2012). Among the existing approaches, DEA is one of the most desirable models that are used in multi-response optimization problems. DEA is a fractional mathematical programming method for measuring the relative efficiency of a set of competitive and homogeneous decision-making units (DMUs) in which multiple inputs and multiple outputs exist (Charnes et al., 1978). On the other hand, in some cases, when a number of control factors and their level increase, TM can lead to a large number of experiments. Sometimes, due to the presence of some inescapable factors, only some of them can be considered resulting in unknown results (Liao, 2004). Indirect experiments are designed with the NN technique, which is a suitable method for estimating other tests. Capability of $\mathrm{NN}$ in complex nonlinear problems makes this method more accurate than other methods such as regression to achieve prediction. Researchers have used the NN tool to solve MRT problems because of its flexibility and ease of use. Gutiérrez and Lozano used an artificial neural network (ANN) to estimate the response for all surface factor compounds (Gutiérrez and Lozano, 2010). In a study on the optimization of parameter parameters of parameters of gas arc parameters, (Lin, 2012) initially used the BPNN to evaluate the graymatter values and then the Levenberg-Marquardt BP (LMBP) algorithm to provide a nonlinear relationship between the parameters Welding and Gray Connection Degree of Welding Machine. Also, Kuo et al. (2012) combined DEA and BPNN to estimate incomplete data and obtain optimal process parameters in a $\mathrm{ZnO}$ : $\mathrm{Al}$ (AZO) laser cutting. In order to facilitate training, three separate NNs with eight neurons are designed as controlled factors in the input layer and a neuron is designed as the ratio of $\mathrm{S} / \mathrm{N}$ in the output layer. In addition, Mondal et al. (2014) used an ANN to predict the signal-to-noise ratio $(\mathrm{S} / \mathrm{N})$ response properties in a $\mathrm{CO} 2$ laser cutting process.

In this paper, an integrated MRT, NN, and RDEA method is presented to find the optimal combination of the parameters of the forwarding device. Initially, due to the factors and their number of levels, an orthogonal oratory of the appropriate Taguchi array (OA) is designed. The OA represents the minimum test needed to observe the effect of factors on two quality attributes, then the $\mathrm{S} / \mathrm{N}$ composition ratio designed to measure the effects of factor levels on responses is computed. In addition, to obtain the effects of factor levels on the response, the $\mathrm{S} / \mathrm{N}$ ratio calculated for training and testing BPNN is used to estimate the entire $\mathrm{S} / \mathrm{N}$ ratio. For simplicity and data usage, the S / N ratio is estimated. Finally, the normalized S / N ratio in the RDEA model is used to obtain the desired combination of the parameters of the forming device.

The Taguchi Fuzzy optimization of the model can be introduced through the Taguchi design and ANOVA to the TFO. The results can help to prove the benefits of parameters related to economic and environmental factors in system benefits, total product quality and quality. Identify the optimal parameters of the parameter to obtain the desired systemic benefits and simultaneously reducing the cycle time. In the MRT method, due to the presence of some uncontrollable factors or the impossibility of carrying out all tests, only some tests were performed and other experiments were designed with the FNN technique. Clearly, in FNN results, there is uncertainty that can lead to inaccurate decisions. Hence, a robust optimization approach is used to address uncertainty in FNN results. In this paper, the experiment was first designed by Taguchi method, then some tests are performed and other experiments are estimated using the BPFNN method. Finally, the best combination of optimal control parameters is selected using FRDEA optimal data envelopment analysis model. Also, S / N and ANOVA methods have been used to study the 
International Journal of Mathematical, Engineering and Management Sciences

Vol. 4, No. 3, 580-590, 2019

https://dx.doi.org/10.33889/IJMEMS.2019.4.3-046

characteristics of process performance, which show that the four control parameters of sheet feeding speed, wind pressure, molding temperature and sheet thickness affect process quality, and the proposed approach is a systematic and efficient method. It reduces the cycle time.

\section{Fuzzy Neural Network}

In a neural network perspective, we have tried to model the functioning of the nervous system and the human brain. This method can solve complex problems by relying on learning ability and parallel processing ability in natural neural networks. The ability of the neural networks has been confirmed with application in various issues of signal processing, modeling, modeling, identification, forecasting, control, and optimization in recent decades. Nowadays, these structures are considered as one of the most common non-models is used. In fuzzy systems, approximate decision-making syntax is based on individual or group human experience. The great advantages of fuzzy systems are their simplicity and comprehensibility, making them very easy to use and understand. To implement the neural network of a fuzzy system, we first define a generic neuron. In general, the input-output relationship is defined as follows

$\mathrm{O}_{i}^{k}=\alpha\left(f\left(U_{1}^{k}, U_{2}^{k}, \ldots, U_{n}^{k}, W_{1}^{k}, W_{2}^{k}, \ldots, W_{n}^{k}\right)\right)$

$f$ The central function of the neuron, $\alpha$ is the activity of the neuron, $U_{i}^{k}$ is the input of the $i$ of the neon layerk, $W_{i}^{k}$ is the input weight $i$, of the $k$-layer, and $\mathrm{O}_{i}^{k}$ the output of the neuron $\mathrm{i}$ is in the $\mathrm{k}$ layer. This network has 5 layers, which is the first layer of the input, respectively, in which the weights of the neurons in this layer are all fixed and equal to one. Therefore, we have this layer.

$f=U_{i}^{1}, \alpha=k f$

$k$ is the coefficient of change in the input scale.

The second layer is the fuzzy layer that we use from the Gaussian derived membership function as follows:

$f=-\frac{\left(U_{i}^{2}-m_{i j}\right)}{\sigma_{i j}}, \alpha=e^{f^{2}}$

In the above equation, $m_{i j}$ and $\sigma_{i j}$ respectively, the center (mean) and the width (standard deviation) of the Gaussian functions are related to the $i$ fuzzy set. By setting them, we set the input membership functions.

The third layer is the fuzzy inference layer, where we have a multiplicative neuron per rule. In this layer, fuzzy rules are obtained.

$f=U_{1}^{3} * U_{2}^{3} * U_{3}^{3} * \ldots * U_{m}^{3} \quad, \alpha=f$

In the fourth layer, the fuzzy rules get normalized.

$f_{i}=U_{i}^{4} \quad, y_{i}=\alpha_{i}=\frac{f_{i}}{\sum_{i=1}^{m} U_{i}^{4}}$ 
International Journal of Mathematical, Engineering and Management Sciences

Vol. 4, No. 3, 580-590, 2019

https://dx.doi.org/10.33889/IJMEMS.2019.4.3-046

In the fifth layer, where the output of the fuzzy neural network is obtained which can be used for other decisions, in some studies, the above method is referred to as non-fuzzy, and in contrast to the non-fuzzy linear term used It is defined for the non-fuzziness of the middle centers. The functions associated with this layer are defined as follows:

$f=\sum_{i=1}^{m} W_{i}^{5} * U_{i}^{5} \quad, y=\alpha=f$

In fact, $U_{i}^{4}, O_{i}^{4}, W_{i}^{4}$ is the center of the fuzzy set of output $i$ and the number of output phase fuzzy sets.

To evaluate the accuracy of the proposed model and to evaluate the results using the experimental data in the adaptive fuzzy neural network, we can use the mean error.

$\% E_{\text {avg }}=\frac{1}{N} \sum_{i=1}^{n}\left(\frac{y_{i}-y_{\text {exp }}}{y_{i}}\right) * 100$

In the above relation, $N$ is the number of samples, $y_{i}$ is the response value generated by the model, and $\% E_{\text {avg }}$ is the average error value that $y_{\text {exp }}$ expresses the corresponding response value of the experiment.

\section{Data Envelopment Analysis}

Data Envelopment Analysis (DEA) is a method for obtaining the relative efficiency of a single decision maker (DMU), in that it compares it with the linear combinations involved with other DMUs. Decision making units are homogeneous units with the same inputs and outputs. Performance measurement has always been a concern for researchers due to its importance in evaluating the performance of an organization. In the groundbreaking work of Farrell, an economist, a nonparametric method was proposed to determine the relative efficiency of units. He outlined a set of empirically monitored decision makers a way to calculate the small levels of efficient production boundaries. Charles and colleagues introduced DEA: A linear programming technique to measure the kind of performance that Farrell described. The model provided by Charles and colleagues was able to measure unit performance with multiple inputs and multiple outputs. This model is one of the basic models in data envelopment analysis, known as the CCR model, in which the efficiency is assumed to be constant. Bunker et al. presented a model with the assumption of return on a variable scale, which became known as the BCC model. DEA application areas have expanded rapidly in recent decades. Model (8) shows a CCR enclosure model of the axle input (Fazli-Khalaf et al., 2017; Hadi-Vencheh and Yousefi, 2018). 
International Journal of Mathematical, Engineering and Management Sciences

Vol. 4, No. 3, 580-590, 2019

https://dx.doi.org/10.33889/IJMEMS.2019.4.3-046

$$
\begin{aligned}
& \text { Min } y_{0}=\theta \\
& \text { st : } \\
& \sum_{j=1}^{n} \lambda_{j} y_{r j} \geq y_{r 0} \quad(r=1,2, \ldots, s)
\end{aligned}
$$

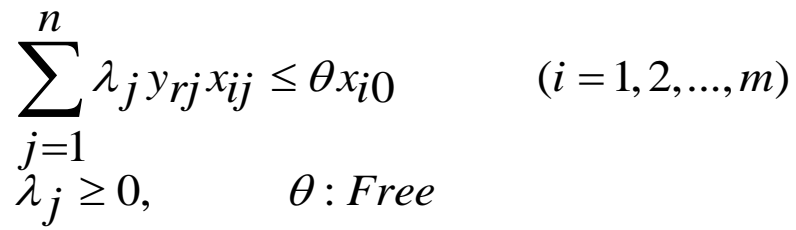

In this paper, we use a non-decreasing return-to-scale (NDRS) axis input model and environmental factors that are defined as follows (9)

$$
\begin{aligned}
& \operatorname{MinZ}_{0}=\theta-\sum_{i=1}^{2} \varepsilon \cdot S n_{i}-\sum_{r=1}^{3} \varepsilon \cdot S p_{r} \\
& \text { s.t : } \\
& \sum_{j=1}^{9} y_{r j} \lambda_{j}-S p_{r}=y_{r_{0}} \quad r=1, \ldots, 5 . \\
& \sum_{j=1}^{9} x_{i j} \lambda_{j}+S n_{i}-\theta \cdot x_{i_{0}}=0 \quad i=1,2,3 . \\
& \sum_{j=1}^{9} E_{l j} \lambda_{j}-\theta . E_{l_{0}} \leq 0 \quad l=1, \ldots, 10 . \\
& \sum_{j=1}^{9} \lambda_{j} \geq 1 \\
& \lambda_{j}, S p_{r}, S n_{i} \geq 0, \theta: \text { Free }
\end{aligned}
$$

\subsection{Robust Data Envelopment Analysis (RDEA)}

In most real-world environments, we often try to describe a supply-side design problem, whose parameters are not known before. We need to take this uncertainty into account when producing a solution, and let's keep in mind that there may be more than one possible occurrence in this problem. In a true decision process, we often encounter a combination of uncertainty, including local and structural uncertainty. We can define a range of possible or sometimes probabilistic or probabilistic distributions for managing uncertainty.

Fazli-Khalaf et al. (2017), described a method of the robust stochastic fuzzy rule that uses fuzzy numbers of triangular LRs and, under the combined uncertainty, with a probability average and a fuzzy scenario, they considered three aspects. 
International Journal of Mathematical, Engineering and Management Sciences

Vol. 4, No. 3, 580-590, 2019

https://dx.doi.org/10.33889/IJMEMS.2019.4.3-046

(i). The average probable value of the objective function's weight. (ii). Possible changeability. (iii). The goal function scenario changes. That incorporates average costs, desirable stability, and reliability in this method.

The proposed model by Fazli-Khalaf et al. (2017) is presented as follows:

$$
\begin{aligned}
\min Z=\left[\frac{C^{(1)}+C^{(2)}+C^{(3)}}{3}\right] x+\sum_{s} P_{s}\left[\frac{d^{(1)}+d^{(2)}+d^{(3)}}{3}\right] y_{s} \\
+\beta \sum_{s} P_{s}\left[\left(\left[\frac{d^{(1)}+d^{(2)}+d^{(3)}}{3}\right] y_{s}\right.\right. \\
\left.\left.\quad-\sum_{s^{\prime}} P_{s^{\prime}}\left[\frac{d^{(1)}+d^{(2)}+d^{(3)}}{3}\right] y_{s^{\prime}}\right)+2 \theta_{s}\right]+\sum_{s} \delta \varepsilon_{s}
\end{aligned}
$$

S. t.

$$
\begin{aligned}
A x \leq[(2 \alpha-1) & \left.b^{(1)}+(2-2 \alpha) b^{(2)}\right] \backslash\left[(2 \eta-1) f^{(1)}+(2-2 \eta) f^{(2)}\right] y_{s}+\varepsilon_{s} \\
& \geq\left[(2 \varphi-1) h_{s}^{(3)} b^{(3)}+(2-2 \varphi) h_{s}^{(2)}\right]\left[\frac{d^{(1)}+d^{(2)}+d^{(3)}}{3}\right] y_{s} \quad \forall s \\
& -\sum_{s} P_{s}\left[\frac{d^{(1)}+d^{(2)}+d^{(3)}}{3}\right] y_{s}+\theta_{s} \geq 0
\end{aligned}
$$

where parameters $c$ and $d$ in the objective function are regarded as uncertain that has triangular possibility distribution and they are formulated based on expected value of uncertain parameters. In the equivalent crisp model, it is assumed that uncertain parameter $c$ has the triangular membership function and could be represented as $C=C^{(1)}+C^{(2)}+C^{(3)}$. Objective function is modeled on the basis of average value of uncertain parameters. Parameters $d, f$ and $h_{s}$ are regarded as uncertain coefficients of constraints and they are modeled regarding satisfaction level of uncertain parameters $0.5<\alpha, \eta, \varphi, \beta \leq 1$. In other words, satisfaction level of each uncertain parameter should be determined based on preference of company DMs. Notably, increasing satisfaction level of uncertain parameters would result in risk-averse output decisions of extended model. The robust DEA (RDEA) model of this paper is formulated as follows:

$\operatorname{Max} Z=\theta_{s 0}$

S.t.

$\begin{aligned} \sum_{s} h_{s} y_{s 0}-\theta_{s 0} & -\sum_{s} P_{s}\left[\frac{d^{(1)}+d^{(2)}+d^{(3)}}{3}\right] y_{s}-(2 \varphi-1) h_{s}^{(3)} b^{(3)} \\ & +(2-2 \varphi) h_{s}^{(2)} \geq 0\end{aligned}$ 
International Journal of Mathematical, Engineering and Management Sciences

Vol. 4, No. 3, 580-590, 2019

https://dx.doi.org/10.33889/IJMEMS.2019.4.3-046

$$
\begin{aligned}
& \sum_{s} f_{s} y_{s 0}-\sum_{s} P_{S}\left[\frac{d^{(1)}+d^{(2)}+d^{(3)}}{3}\right] y_{s} \\
& -\left[(2 \alpha-1) b^{(1)}+(2-2 \alpha) b^{(2)}\right] \quad \forall s^{\prime} \\
& \backslash\left[(2 \eta-1) f^{(1)}+(2-2 \eta) f^{(2)}\right]+\varepsilon_{s} \geq 0 \\
& x, \varepsilon_{s}, y_{s} \geq 0,0.5<\alpha, \eta, \varphi, \beta \leq 1
\end{aligned}
$$

where $f_{s}$ and $h_{s}$ is the $r$ th output for company, $y_{s 0}$ and $\theta_{s 0}$ refer to $r^{\text {th }}$ output and efficiency score of DMU under consideration.

\section{Industrial Case of Forming Salon}

Forming machines have a setup time to get started. When to prepare the machine for the start of the production process must be considered. The materials of the forming salon are polystyrene sheets produced by the extrusion salon, and the final product of the forming salon is the plates produced during Thermoforming operations in this salon. Each forming machine is individually a line and each machine is switched on or off, regardless of whether other Forming machines are turned on or off. The actual production volume of the forming salon depends on the request of the printing salon. In other words, since the final product of the forming salon should be used as a raw material in the printing salon, white glasses should be printed, so the shutting down or turning on of the forming machines depends on the demand for the printing salon. In addition to item 2, if the extruder room was not able to provide the sheet needed for any reason, and the warehouse sheet does not meet the needs of the salon, forging machines will be turned off due to lack of sheets.

Steps for the proposed approach to address uncertainty in the BPNN results are impossible to estimate the experiments. And obtaining the best levels of factors in MRT problems is as follows:

Step 1: Use a standard array of Taguchi standard arrays according to the selected parameters and their surface, the complex and nonlinear effects of the parameters on the quality of reaction and interaction properties Effects of Parameters This step is related to DOE TM. Also, the appropriate formula is based on the $\mathrm{S} / \mathrm{N}$ ratio the aim of the study is to increase, decrease or maintain the value of qualitative characteristics.

Step 2: Use BPNN to create a relationship between model input and output. Number of entries represents a number of control parameters of the model and the number of outputs equal to a few $\mathrm{S} / \mathrm{N}$ ratio of response. A significant number of hidden neurons of layers are often set up using experiment and error and based on it Indicators such as Square Mean Error (MSE) or Linear Square Mean Error (RMSE). Lower the MSE and RMSE indicators mean that the designed network brings the $\mathrm{S} / \mathrm{N}$ ratio closer to real values.

Step3: In order to reduce errors and deal with the problem of different units, normalize FNN results.

Step 4: Apply the RDEA model to calculate the relative efficiency of each test and handle uncertainty about it. FNN results In the RDEA model, FNN, S / N are the normal ratio as the output variables. 
International Journal of Mathematical, Engineering and Management Sciences

Vol. 4, No. 3, 580-590, 2019

https://dx.doi.org/10.33889/IJMEMS.2019.4.3-046

Step 5: Take any test (DMU) based on the relative efficiency measured in step 4 and get the best result.

Selection of control parameters and their levels a typical forgiving cycle described in the previous section is therefore the selected control parameters as follows:

- Feed rate: The advance speed of the extruder plate production plate into the forging machine, which is characterized by R14

- Wind pressure: The amount of wind pressure in the sheet produced from the extruder in the forging machine, which is characterized by R15.

- Sheet thickness: The plate fluctuation generated from the extruder housings in the forging machine, which is characterized by R16.

- Molding temperature: Molding temperature in the forming machine, which is characterized by R17.

The control parameters are variable in the range and their number is shown in Table 1.

Table 1. Control parameters in the domain

\begin{tabular}{|c|c|c|c|c|c|c|}
\hline S.No & Symbol & Control parameters & Range & Level 1 & Level 2 & Level 3 \\
\hline 1 & R14 & Feed speed & $30-18$ & 18 & 22 & 30 \\
\hline 2 & R15 & wind pressure & $350-250$ & 250 & 270 & 350 \\
\hline 3 & R16 & sheet thickness & $16-8$ & 8 & 12 & 16 \\
\hline 4 & R17 & Mold temperature & $220-150$ & 150 & 220 & - \\
\hline
\end{tabular}

The results of the efficiency score of each combination of parameters are given in Table 2. In the RDEA model, all restrictions must be set. Because in this model, the data of two outputs are uncertain, to ensure full protection for all constraints is equal to 2. The typical DEA results are also listed in Table 2 to see how uncertainty can affect relative performance scores.

Table 2. The results of DEA and RDEA models

\begin{tabular}{|c|c|c|c|c|}
\hline Trial no. & $D E A$ & $E 1$ & E2 & E3 \\
\hline $\mathrm{T} 1$ & 0.982 & 0.94349 & 0.8884 & 0.8365 \\
\hline T5 & 0.84 & 0.8070 & 0.76 & 0.7155 \\
\hline T7 & 1 & 0.9642 & 0.9047 & 0.8518 \\
\hline T9 & 0.914 & 0.8745 & 0.8269 & 0.7785 \\
\hline T13 & 0.580 & 0.5624 & 0.5254 & 0.4947 \\
\hline T17 & 0.928 & 0.8963 & 0.8396 & 0.7905 \\
\hline $\mathrm{T} 21$ & 0.14 & 0.7638 & 0.7192 & 0.6772 \\
\hline $\mathrm{T} 25$ & 0.094 & 0.1345 & 0.1266 & 0.1192 \\
\hline T29 & 0.036 & 0.0909 & 0.0856 & 0.0806 \\
\hline T33 & 0.006 & 0.6996 & 0.0325 & 0.0606 \\
\hline T37 & 0.296 & 0.1426 & 0.0052 & 0.0051 \\
\hline T41 & 1 & 0.0057 & 0.2653 & 0.8564 \\
\hline
\end{tabular}


International Journal of Mathematical, Engineering and Management Sciences

Vol. 4, No. 3, 580-590, 2019

https://dx.doi.org/10.33889/IJMEMS.2019.4.3-046

To predict unfinished experiments, Liao used BPNN. However, the uncertainty was considered in the BPNN results and the relative performance scores were calculated using the usual DEA. In this section, relative performance scores for DMUs are recalculated with regard to uncertainty in predicted data, and the results of the DEA and RDEA models are presented in Table 3.

Table 3. Comparison the proposed approach

\begin{tabular}{|c|c|c|c|c|c|}
\hline $\boldsymbol{D M U}$ & $\boldsymbol{D E A}$ & $\boldsymbol{R D E A}$ & $\boldsymbol{D M U}$ & $\boldsymbol{D E A}$ & $\boldsymbol{R} \boldsymbol{A E A}$ \\
\hline 1 & 96.63 & 83.99 & 13 & 100 & 83.95 \\
\hline 2 & 98.46 & 80.85 & 14 & 99.96 & 85.92 \\
\hline 3 & 100 & 85.35 & 15 & 99.82 & 85.52 \\
\hline 4 & 96.03 & 85.33 & 16 & 99.11 & 85.51 \\
\hline 5 & 97.90 & 84.57 & 17 & 99.97 & 94.36 \\
\hline 6 & 100 & 85.56 & 18 & 98.43 & 76.08 \\
\hline 7 & 95.63 & 85.64 & 19 & 98.96 & 75.37 \\
\hline 8 & 97.63 & 89.10 & 20 & 97.63 & 78.85 \\
\hline 9 & 100 & 83.95 & 21 & 100 & 84.25 \\
\hline 10 & 98.22 & 94.36 & 22 & 98.55 & 87.15 \\
\hline 11 & 98.96 & 83.67 & 23 & 99.23 & 94.36 \\
\hline 12 & 99.18 & 85.10 & 24 & 100 & 85.33 \\
\hline
\end{tabular}

As shown in Table 3, 6 factors / compound levels with performance rates of 100.00 (high red) are selected as an optimal combination for improving product quality. Using the proposed RDEA model, performance scores are calculated. Table 3 shows that numbers 17, 23 and 10 are selected (highlighted in blue). The results show that considering the disturbance in NN results can lead to the selection of the new optimization. It is clear that there is uncertainty in the output of $\mathrm{NN}$ and is necessary for uncertainty in NN results. As already mentioned, the efficacy values produced by the RDEA have safety limitations relative to the disorder below the DEA model. The results show that the proposed approach is reliable and applicable to the manufacturing industry of disposable containers.

Decision makers and experts in the quality control sector, with allowance for criteria such as cost, can choose the parameter setting among the efficient sets for the Forming Machine. Given the uncertainty in the data, the typical DEA method is uncertain and may help to make a mistake. After applying RDEA, the results of the proposed method are shown in Table 3 and the sensitivity analysis of the RDEA model is considered to be different values of $0.02,0.05$ and 0.08. RDEA results are calculated for all trials. The results indicate that, as higher values, performance scores naturally result in lower immunity constraints to fewer disturbances.

\section{Conclusion}

This paper describes a Taguchi method for optimizing control parameters in the forming process. Experimental results show that the cycle time for a forming process can be reduced simultaneously. Regarding the results, the lack of attention to uncertainty in the use of linear programming may result in unreliable and inaccurate results. In addition, with increasing data impairment, RDEA results in a safety model relative to uncertainty performed much better. For future studies, readers can apply stochastics disturbances to RDEA data and compare results with the proposed model. 
International Journal of Mathematical, Engineering and Management Sciences

Vol. 4, No. 3, 580-590, 2019

https://dx.doi.org/10.33889/IJMEMS.2019.4.3-046

\section{Conflict of Interest}

The author(s) confirm that this article contents have no conflict of interest.

\section{Acknowledgement}

Authors appreciate the reviewers and the respected editor of IJMEMS for their constructive comments leading to strengthen the quality and presentation of the paper.

\section{References}

Antony, J. (2001). Simultaneous optimisation of multiple quality characteristics in manufacturing processes using Taguchi's quality loss function. The International Journal of Advanced Manufacturing Technology, 17(2), 134-138.

Charnes, A., Cooper, W.W., \& Rhodes, E. (1978). Measuring the efficiency of decision making units. European Journal of Operational Research, 2(6), 429-444.

Fazli-Khalaf, M., Mirzazadeh, A., \& Pishvaee, M.S. (2017). A robust fuzzy stochastic programming model for the design of a reliable green closed-loop supply chain network. Human and Ecological Risk Assessment: An International Journal, 23(8), 2119-2149.

Fisher, R.A., \& Yates, F. (1943). Statistical tables for biological, agricultural and medical research. Oliver and Boyd Ltd, London.

Gutiérrez, E., \& Lozano, S. (2010). Data envelopment analysis of multiple response experiments. Applied Mathematical Modelling, 34(5), 1139-1148.

Hadi-Vencheh, A., \& Yousefi, A. (2018). Selecting six sigma project: a comparative study of DEA and LDA techniques. International Journal of Lean Six Sigma, 9(4), 506-522.

Huang, M.L., Hung, Y.H., \& Yang, Z.S. (2016). Validation of a method using Taguchi, response surface, neural network, and genetic algorithm. Measurement, 94, 284-294.

Kuo, C.F.J., Vu, H.Q., Gunawan, D., \& Lan, W.L. (2012). Multi-objective optimization of laser-scribed micro grooves on AZO conductive thin film using data envelopment analysis. Optics \& Laser Technology, 44(6), 1959-1970.

Liao, H.C. (2004). A data envelopment analysis method for optimizing multi-response problem with censored data in the Taguchi method. Computers \& Industrial Engineering, 46(4), 817-835.

Lin, H.L. (2012). The use of the Taguchi method with grey relational analysis and a neural network to optimize a novel GMA welding process. Journal of Intelligent Manufacturing, 23(5), 1671-1680.

Madhavi, S.K., Sreeramulu, D., \& Venkatesh, M. (2017). Evaluation of optimum turning process of process parameters using DOE and PCA Taguchi Method. Materials Today: Proceedings, 4(2), 19371946.

Mondal, S., Bandyopadhyay, A., \& Pal, P.K. (2014). Application of artificial neural network for the prediction of laser cladding process characteristics at Taguchi-based optimized condition. The International Journal of Advanced Manufacturing Technology, 70(9-12), 2151-2158.

Muthuramalingam, T., \& Mohan, B. (2014). Application of Taguchi-grey multi responses optimization on process parameters in electro erosion. Measurement, 58, 495-502.

Nian, C.Y., Yang, W.H., \& Tarng, Y.S. (1999). Optimization of turning operations with multiple performance characteristics. Journal of Materials Processing Technology, 95(1-3), 90-96. 
International Journal of Mathematical, Engineering and Management Sciences

Vol. 4, No. 3, 580-590, 2019

https://dx.doi.org/10.33889/IJMEMS.2019.4.3-046

Pandey, R.K., \& Panda, S.S. (2014). Optimization of bone drilling parameters using grey-based fuzzy algorithm. Measurement, 47, 386-392.

Rao, D.B., Rao, K.V., \& Krishna, A.G. (2018). A hybrid approach to multi response optimization of micro milling process parameters using Taguchi method based graph theory and matrix approach (GTMA) and utility concept. Measurement, 120, 43-51.

Sastry, M.N.P., Devi, K.D., \& Reddy, K.M. (2012). Analysis and optimization of machining process parameters using design of experiments. Industrial Engineering Letters, 2(9), 23-32.

Sibalija, T.V., \& Majstorovic, V.D. (2012). An integrated approach to optimise parameter design of multiresponse processes based on Taguchi method and artificial intelligence. Journal of Intelligent Manufacturing, 23(5), 1511-1528. 